\title{
Complete Sternal Cleft Repair
}

\author{
Ankita Harijee $\quad$ Sundeep Vijayaraghavan ${ }^{1}$ Arjun Reddy Marathi ${ }^{1}$ Brijesh Parayaru Kottayil ${ }^{2}$ \\ Mahesh Kappanayil $^{3}$ Praveen Reddy Bayya² Jessin P Jayashankar ${ }^{4}$
}

\author{
${ }^{1}$ Department of Plastic \& Reconstructive Surgery, Amrita Institute \\ of Medical Sciences \& Research Centre, Kochi, India \\ 2Department of Cardiovascular \& Thoracic Surgery, Amrita Institute \\ of Medical Sciences \& Research Centre, Kochi, India \\ ${ }^{3}$ Department of Paediatric Cardiology, AIMS 3D Printing \& \\ Innovation Laboratory, Amrita Institute of Medical Sciences \& \\ Research Centre, Kochi, India \\ ${ }^{4}$ Department of Anesthesiology, Amrita Institute of Medical \\ Sciences \& Research Centre, Kochi, India
}

Indian J Plast Surg:2020;53:419-422

\author{
Address for correspondence Sundeep Vijayaraghavan, MS, \\ MCh, DNB, Department of Plastic \& Reconstructive Surgery, \\ Amrita Institute of Medical Sciences, Kochi, Kerala, 682041, India \\ (e-mail: veesundeep@gmail.com).
}

\begin{abstract}
Keywords

- 3D printing

- chest wall reconstruction

- sternal cleft

Sternal cleft (SC) is a rare congenital malformation which can be partial or complete. We report a case of complete SC in a 9-month-old child. Our technique involves a combination of reinforcement with the deep cervical fascial extension, followed by the anterior perichondrial flaps, bridged with the rib graft, incorporating surplus resected cartilaginous xiphoid process, and covered with the bilateral pectoralis major muscle flap for the chest wall reconstruction with 3D printing assisting preoperative planning. The size of the defect in relation to the age of presentation was a deciding factor in the adoption of this alternative surgical technique.
\end{abstract}

\section{Introduction}

Complete sternal cleft (SC) is an uncommon congenital chest wall malformation. Chest wall reconstruction provides protection to the mediastinal structures, additionally aiding in the restoration of the aesthetic appearance.

\section{Case Report}

A 9-month-old female child born out of nonconsanguineous marriage reported to us with a history of visible pulsations in the chest. On clinical evaluation, a $5 \times 5 \mathrm{~cm}$ wide, "V"-shaped complete SC was noted converging toward the xiphisternum with visible mediastinal pulsation ( - Fig. 1). Clavicles were $5 \mathrm{~cm}$ apart with medial ends floating. There was an associated midline contracture band in the neck which restricted complete neck extension. Supraumbilical midline raphe was present.

Contrast-enhanced CT chest was suggestive of the absence of midline fusion of sternum, with the presence of the xiphoid process ( - Fig. 2 ). US abdomen was within normal limits. Two-dimensional (2D) echocardiogram showed a $8 \mathrm{~mm}$ ostium secundum atrial septal defect (ASD) with left to right shunt. Genetics workup was done to rule out syndromic (pentalogy of Cantrell and posterior fossa malformations, hemangioma, arterial anomalies, coarctation of the aorta/ cardiac defects, and eye abnormalities [PHACE] syndrome) association.

\section{D Printing}

$3 \mathrm{D}$ printing of the available rib cage and the costal cartilage was done in a 3D printing laboratory within our institution. Materialise Mimics InPrint software was used to segment the region. Ribs and spine were printed in polylactic acid (PLA) material using fusion deposition modelling (FDM) printer (Ultimaker 3), and cartilage was separately printed using clear resin material on a sterolithography printer (Formlabs Form2), creating a realistic 3D model. Additionally, the

\section{published online} December 30, 2020
DOI https://doi.org/ 10.1055/s-0040-1721547 ISSN 0970-0358.
C 2020. Association of Plastic Surgeons of India.

This is an open access article published by Thieme under the terms of the Creative Commons Attribution-NonDerivative-NonCommercial-License, permitting copying and reproduction so long as the original work is given appropriate credit. Contents may not be used for commercial purposes, or adapted, remixed, transformed or built upon. (https://creativecommons.org/licenses/by-nc-nd/4.0/).

Thieme Medical and Scientific Publishers Pvt. Ltd. A-12, 2nd Floor, Sector 2, Noida-201301 UP, India 


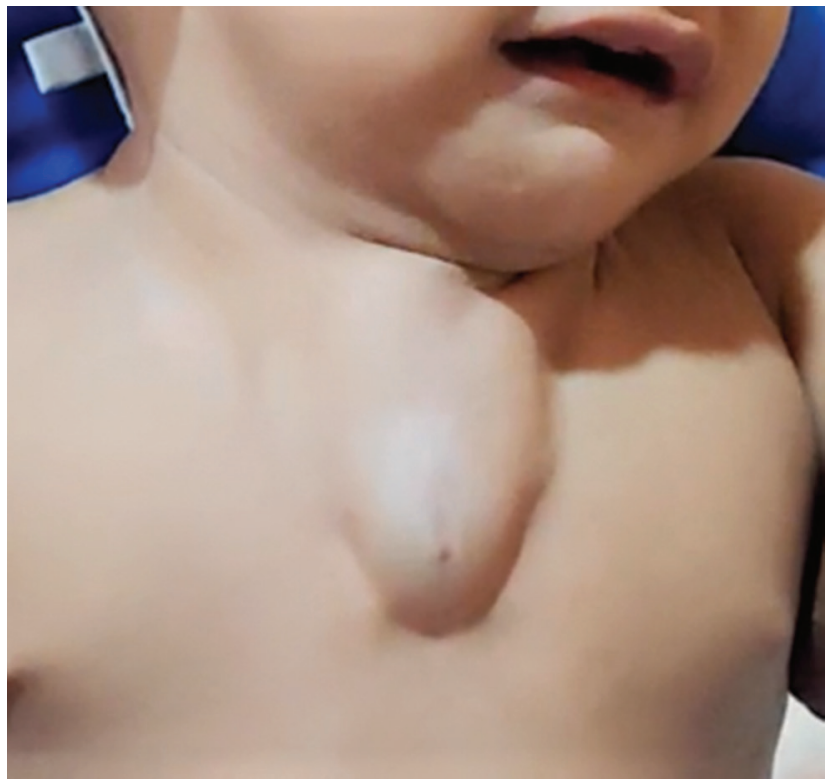

Fig. 1 Image showing "V"-shaped complete sternal cleft with visible pulsation covered by skin.

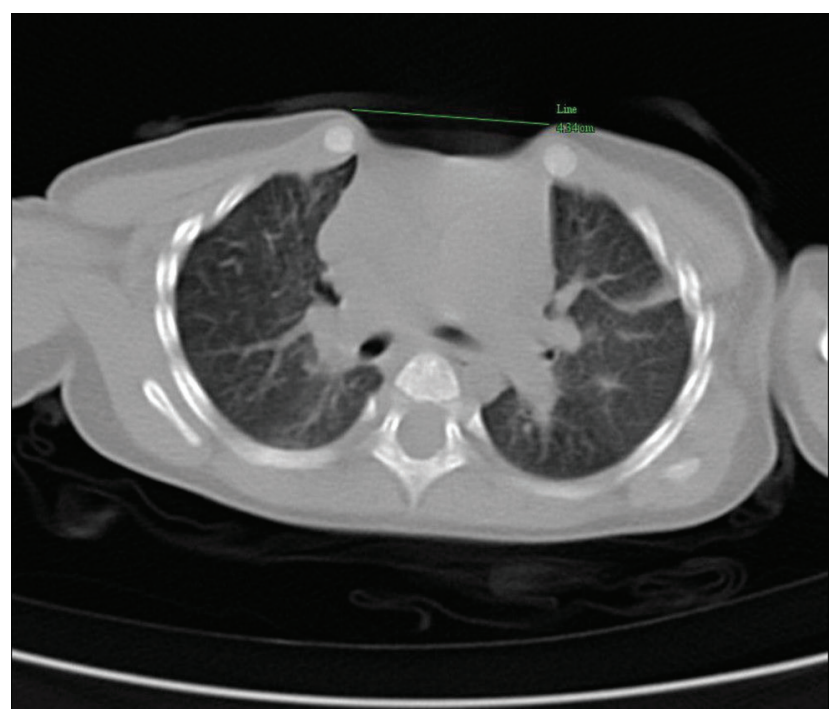

Fig. 2 Image displaying axial section of CECT thorax displaying $5 \mathrm{~cm}$ of sternal cleft between the two medial ends of clavicle.

7th and 8th ribs were printed (-Fig. 3). Incisions of the sternum for bringing the rib cage together were decided on the model. The site, direction, length of incision, and additional rib graft length were preplanned, resulting in minimal incisional approach. To get the proper contour of the upper anterior chest wall, it was decided that $4 \mathrm{~cm}$ of the 7 th rib from right side had to be harvested just anterior to the anterior axillary line.

\section{Surgical Technique}

Midline chest incision was placed, and the skin flaps were raised off the precordium. A $5 \times 5 \mathrm{~cm}$ wide "V"-shaped complete SC was visualized. The deep cervical fascia was seen extending from the neck along the inner aspect of the sternal

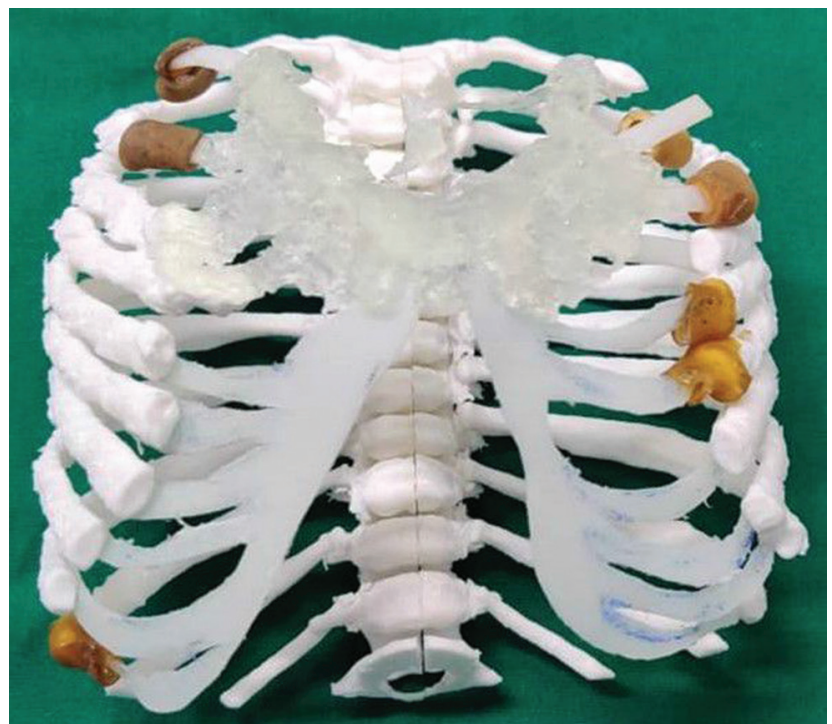

Fig. 3 Image showing 3D-printed model of rib cage with costal cartilage.

cleft edges till the inferior limit of the cleft. Herniation of mediastinal contents through the defect in the extended deep cervical fascia was seen. The fascia was found to be tough and of good quality and strength. Inferiorly xiphoid process was well-formed. Bilateral pectoralis major muscle flap (left side was islanded) was raised.

The anterior perichondrium on either side of the 1 st to 4th costal cartilage was raised like a perichondrial flap from the costochondral junction till the edge of SC. The xiphoid process bone block was excised. In the lower part of sternum, the cartilage was incised in a straight line. The edges were approximated in an overriding fashion and $10 \times 15 \mathrm{~mm}$ cartilage was excised without causing respiratory compromise. Central venous pressure (CVP) (7-8 mm of $\mathrm{Hg}$ ) and $\mathrm{pO}_{2}$ (110-120 $\mathrm{mm}$ of $\mathrm{Hg}$ ) were maintained in the overriding position for 5 minutes. Thereafter, the lower edges of the sternum were primarily approximated using No.1 stainless steel sternal wires. The size of the cleft in the upper sternum was reduced to $4 \mathrm{~cm}$.

Herniated mediastinal contents were reduced by approximating the deep cervical fascia in two layers, with 2-0 ticron continuous sutures. The perichondrial flaps were reflected medially and approximated in crisscross fashion over the deep cervical fascia (-Fig. 4). A $5 \mathrm{~cm}$ graft of right seventh rib was harvested through a separate incision, just medial to the anterior axillary line. The $4 \mathrm{~cm}$ upper sternal defect was bridged using a rib graft with No.1 stainless steel sternal wires (-Fig. 5). The residual defect was filled with xiphoid process bone block and diced cartilage. Bilateral pectoralis major muscle flap was advanced and approximated in the midline using 2-0 ticron. Skin was closed with 4-0 monocryl subcuticular sutures. Postoperatively, the child was on ventilator support for 3 days and discharged on the 10th postoperative day. Follow-up at 1 year reveals no complications with acceptable cosmesis (-Figs. 6 and 7 and - Video 1). 


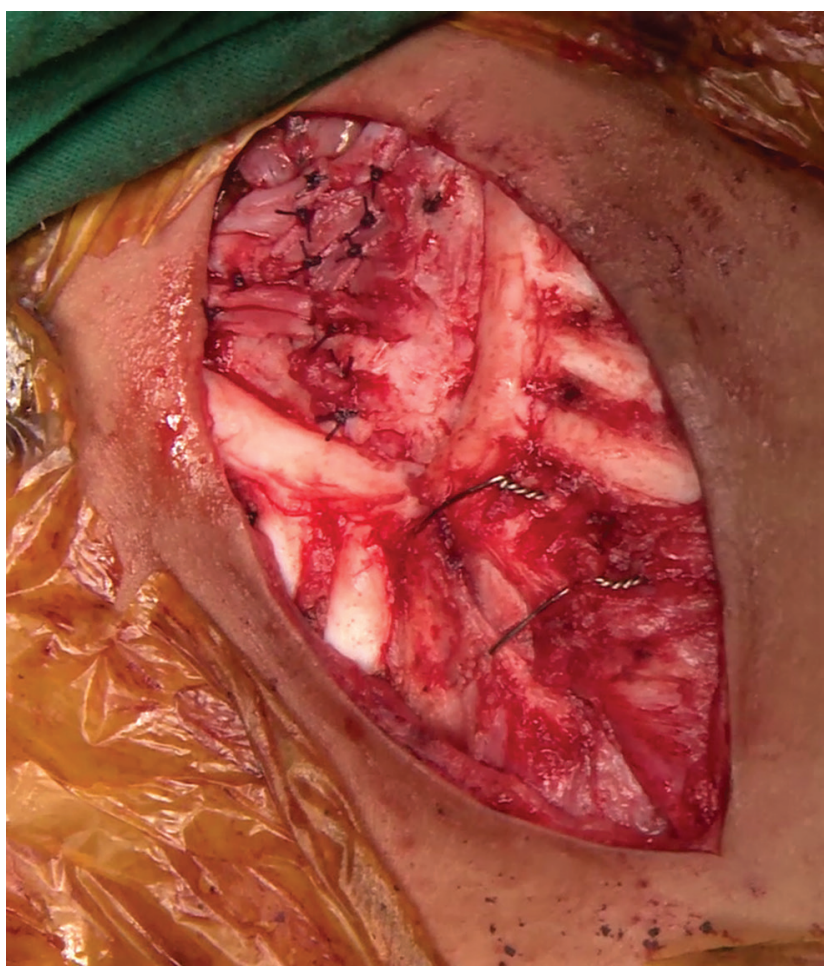

Fig. 4 Intraoperative image showing anterior perichondrial flap from 1 st to 4 th rib was raised on either side and sutured over the sternal defect in a crisscross fashion bridging the defect and primary approximation of lower portion of sternum.

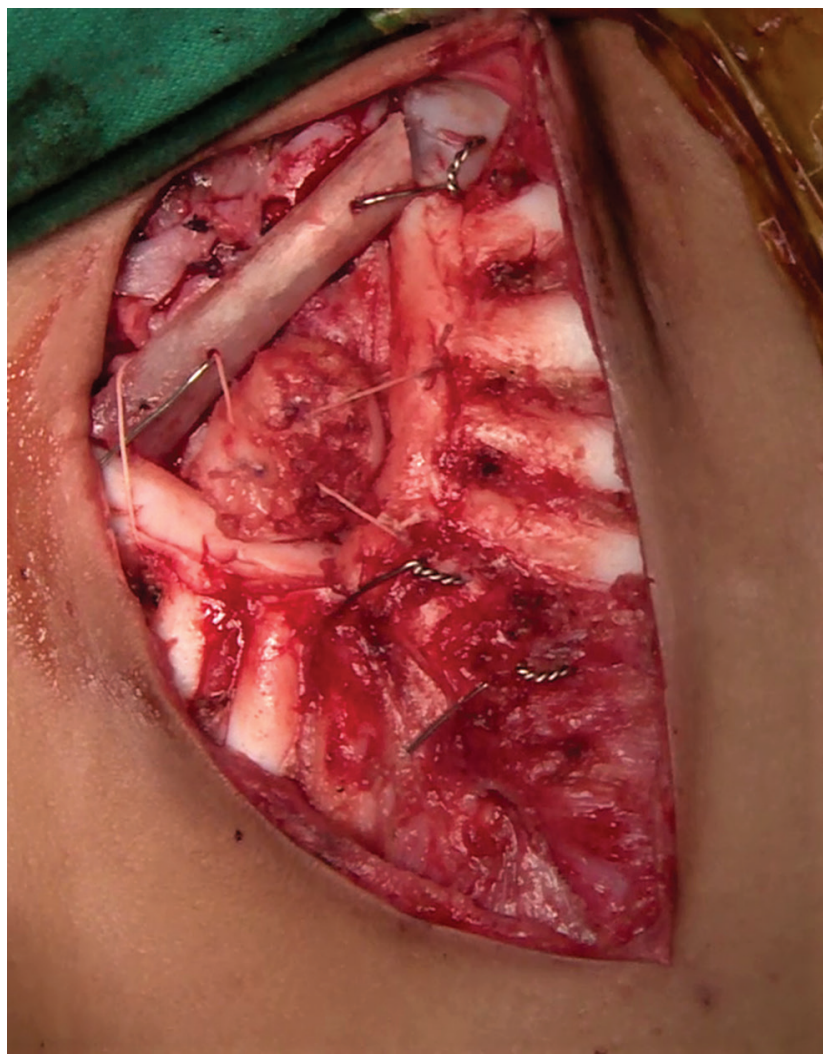

Fig. 5 Intraoperative image showing bridging of upper sternal defect with $4 \mathrm{~cm}$ 7th rib graft and xiphoid bone block interspersed with diced cartilage.

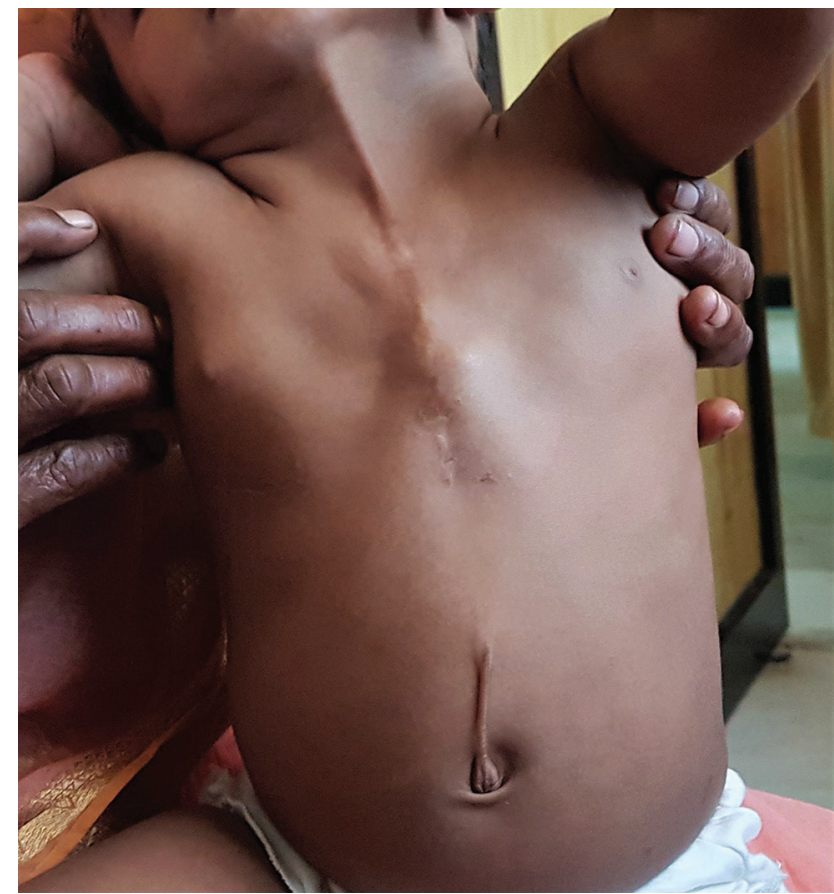

Fig. 6 Postoperative image showing no visible pulsation at 1-year follow-up.

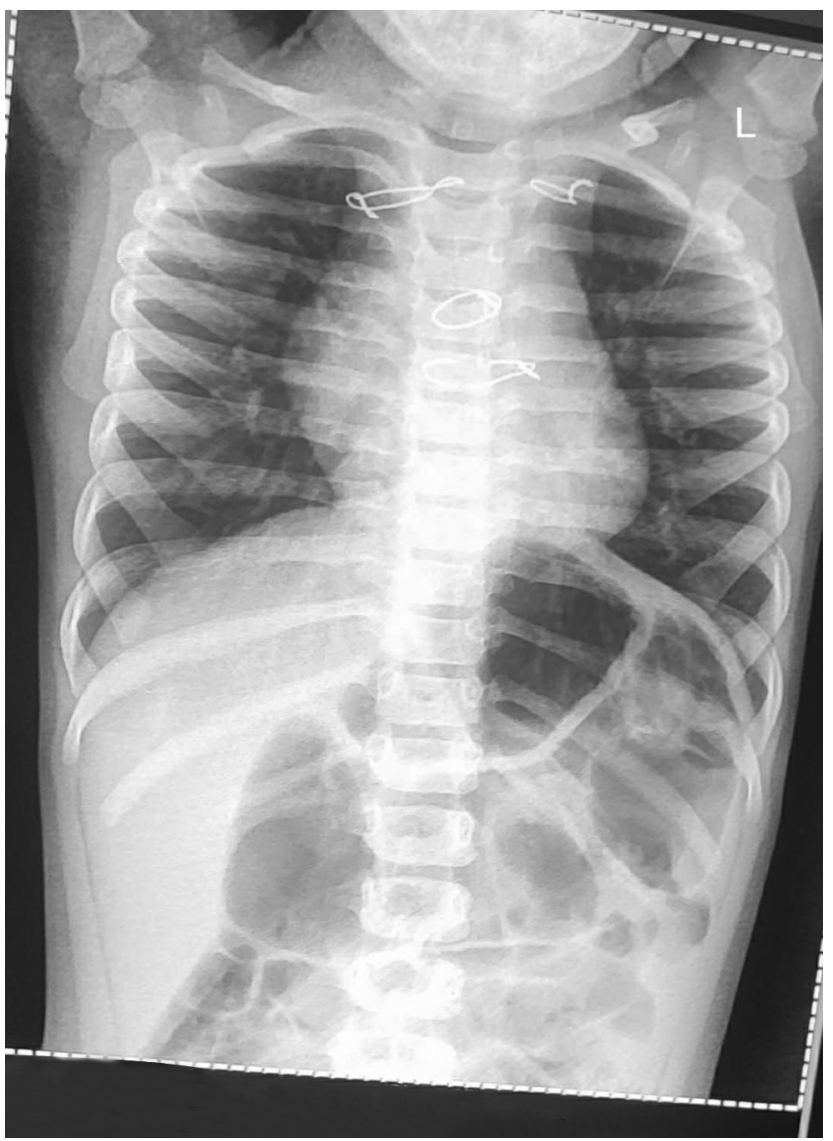

Fig. 7 X-ray at 1-year follow-up showing osseointegration of rib graft with xiphoid process bony block. 


\section{Video1}

Video displaying visible pulsation during expiration and V-shaped sternal cleft (SC) during inspiration with supraumbilical raphe and postoperative outcome. Online content including video sequences viewable at: https://www. thieme-connect.com/products/ejournals/html/10.1055/s-00401721547.

\section{Discussion}

SC can be classified as complete or partial, based on the extent of absence of sternum. ${ }^{1}$ Complete SC can be "U"- or "V"-shaped.

In a "U"-shaped cleft, sternal bars are parallel to each other, which is managed easily by primary approximation. In a "V"-shaped cleft, sternal bars are converging inferiorly; hence, primary approximation becomes difficult without the excision of the bridging segment (-Algorithm 1).

There are several surgical approaches described such

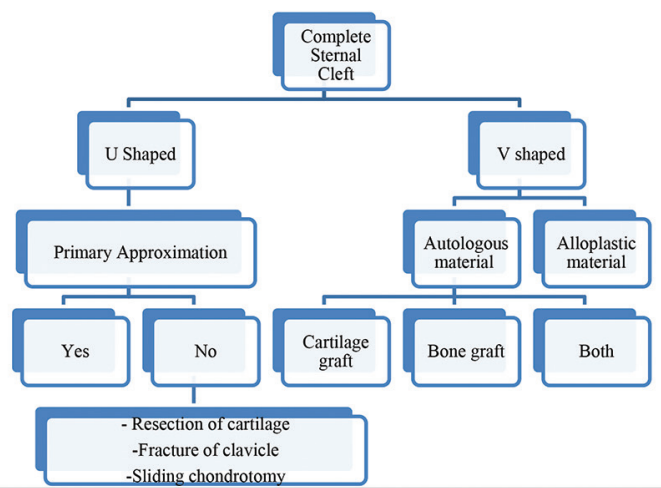

Algorithm 1 for surgical approaches for complete sternal cleft (SC).

as primary closure, closure using autogenous material (cartilage/bone graft/muscle interposition), prosthetic materials, and biological tissue grafts. Primary closure can be achieved, owing to the chest wall elasticity in infants less than 3 months of age, without causing respiratory compromise. As the age progresses, the rigidity of chest wall and the physiologic accommodation of the intrathoracic organs limits the primary approximation, which is facilitated by resection of costal cartilage/fracturing the clavicle or sliding chondrotomy. Prosthetic reconstruction carries risk of extrusion, infection and lacks growth with child, contrary to autologous material. ${ }^{2,3}$

Kabiri et al and ${ }^{4}$ Sapiri et $\mathrm{al}^{5}$ reported complete SC in adolescence and adults associated with pectus excavatum, where resection of the deformed cartilage for correction of pectus excavatum facilitated primary SC approximation.

Biswas et al reported a case of 7-year-old with $4 \times 6 \mathrm{~cm}$ "V"-shaped defect, which was reconstructed with a split iliac bone graft and sternocostal portion of pectoralis major muscle flap. ${ }^{6}$ Jadhav et al reported a case of 12 -year-old with 2.8 to
$3.1 \mathrm{~cm}$ "U"-shaped defect, which was corrected with anterior perichondrial flap and pectoralis major advancement flap. ${ }^{7}$ Jabbad et al repaired a 6-cm defect in a 35-year-old with titanium plates. ${ }^{8}$

In our case, the correction was done in four layers, using the deep cervical fascia, the anterior perichondrial flap with the autologous rib graft, and the bilateral pectoralis major muscle flap. Considering the age of the child ( 9 months), and the size and shape of the defect ("V"-shaped, $5 \mathrm{~cm}$ at upper end), which was wide for the age of the child, primary closure would increase intrathoracic pressure; hence, the alternative repair was done. 3D printing provided additional aid with its features of virtual image of the operative site, preplanning, assistance in counselling and reducing operative duration.

Age at presentation for the management has been described in the literature as preliminary factor in choosing the appropriate surgical technique. ${ }^{3}$ But none of the published literature emphasizes on the defect size in relation to the age of presentation as the decisive factor in determining the surgical technique.

\section{Conclusion}

Besides the age at presentation, the size and shape of the defect in relation to the age can be considered as an additional factor in deciding the appropriate surgical technique for repair in complete SC.

\section{Funding}

No source of funding.

\section{Conflict of Interest}

All the authors declare that there are no conflicts of interest regarding the publication of this paper.

\section{Acknowledgments}

Nil.

\section{References}

1 Ravitch MM, Disorders of the sternum and the thoracic wall. In: Sabiston DC Jr, Spencer FC, eds. Gibbon's Surgery of the Chest. 4th ed. Philadelphia: WB Saunders; 1983:318-360

2 Kamal Y. Sternal cleft: appropriate approach to diagnosis and treatment. Surgery \& Case Studies: Open Access Journal 2018; (e-pub ahead of print). doi: 10.32474/scsoaj.2018.01.000112

3 Firmin RK, Fragomeni LS, Lennox SC. Complete cleft sternum. Thorax 1980;35(4):303-306

4 Kabiri H, Traibi A, Boulahya A. Complete sternal cleft in an adult: case report. Gen Thorac Cardiovasc Surg 2011;59(8):587-589

5 Sarper A, Oz N, Arslan G, Demircan A. Complete congenital sternal cleft associated with pectus excavatum. Tex HeartInstJ2002; 29(3):206-209

6 Biswas G, Khandelwal NK, Venkatramu NK, Chari PS. Congenital sternal cleft. Br J Plast Surg 2001;54(3):259-261

7 Jadhav V, Rao S, D'Cruz A. Autologous repair of isolated complete sternal cleft in an adolescent. J Pediatr Surg 2009;44(12): 2414-2416

8 Jabbad H, Shehata R, Al-Ebrahim KE. Primary repair of complete sternal cleft in an adult: a 4-year follow-up. Tex Heart Inst J 2011;38(4):452-453 